\title{
Twelve-month outcomes of a randomized trial of a moderate-carbohydrate versus very low-carbohydrate diet in overweight adults with type 2 diabetes mellitus or prediabetes
}

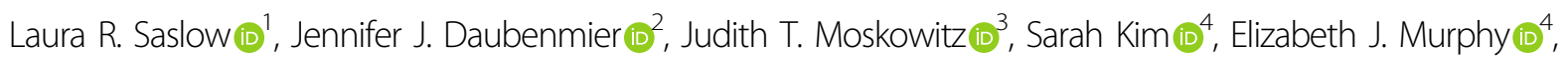

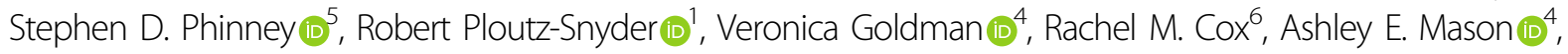
Patricia Moran (10) ${ }^{4}$ and Frederick M. Hecht (iD ${ }^{4}$

\begin{abstract}
Dietary treatment is important in management of type 2 diabetes or prediabetes, but uncertainty exists about the optimal diet. We randomized adults $(n=34)$ with glycated hemoglobin $(\mathrm{HbA} 1 \mathrm{c})>6.0 \%$ and elevated body weight $(\mathrm{BMI}>25)$ to a very low-carbohydrate ketogenic (LCK) diet $(n=16)$ or a moderate-carbohydrate, calorie-restricted, lowfat (MCCR) diet $(n=18)$. All participants were encouraged to be physically active, get sufficient sleep, and practice behavioral adherence strategies based on positive affect and mindful eating. At 12 months, participants in the LCK group had greater reductions in HbA1c levels (estimated marginal mean $(E M M)$ at baseline $=6.6 \%$, at 12 mos $=6.1 \%$ ) than participants in MCCR group (EMM at baseline $=6.9 \%$, at 12 mos $=6.7 \%$ ), $p=.007$. Participants in the LCK group lost more weight (EMM at baseline $=99.9 \mathrm{~kg}$, at $12 \mathrm{mos}=92.0 \mathrm{~kg}$ ) than participants in the MCCR group (EMM at baseline $=97.5 \mathrm{~kg}$, at $12 \mathrm{mos}=95.8 \mathrm{~kg}), p<.001$. The LCK participants experienced larger reductions in diabetes-related medication use; of participants who took sulfonylureas or dipeptidyl peptidase-4 inhibitors at baseline, 6/10 in the LCK group discontinued these medications compared with $0 / 6$ in the MCCR group ( $p=.005$ ). In a 12-month trial, adults with elevated HbA1c and body weight assigned to an LCK diet had greater reductions in HbA1c, lost more weight, and reduced more medications than those instructed to follow an MCCR diet.
\end{abstract}

\section{Introduction}

Nutritional management is an important component in the treatment of type 2 diabetes. Considerable uncertainty exists, though, about the optimal level of carbohydrate intake. Previous research suggests that an ad libitum very low-carbohydrate ketogenic diet (LCK) may improve metabolic measures in adults with type 2

Correspondence: Laura R Saslow (saslowl@umich.edu)

'University of Michigan, Ann Arbor, MI, USA

${ }^{2}$ San Francisco State University, San Francisco, CA, USA

Full list of author information is available at the end of the article diabetes $^{1-6}$ and reduce the need for diabetes-related medications $^{7-10}$.

We randomized adults with type 2 diabetes or prediabetes and elevated body weight to receive an LCK diet or a moderate-carbohydrate, calorie-restricted, low-fat diet (MCCR). We previously reported the initial 3-month outcomes, which showed that participants in the LCK group had significantly better glycemic control and a trend toward greater weight loss compared to participants in the MCCR group ${ }^{11}$. Here we report the outcomes of the trial at 6 and 12 months after baseline. 


\section{Methods}

\section{Study design}

We conducted a parallel-group randomized (1:1) trial. The University of California, San Francisco (USCF) Institutional Review Board approved of this trial, which is registered with ClinicalTrials.gov (NCT01713764). Eligibility criteria included being aged 18 or older, overweight (body mass index (BMI) of 25 or above), with a current glycated hemoglobin $\left(\mathrm{HbA}_{1 \mathrm{c}}\right)$ level over $6.0 \%$. We excluded participants who were currently using insulin or taking more than three glucose-lowering agents. We obtained an informed consent from all eligible participants.

\section{Intervention}

Participants attended 19 classes over 12 months: Twelve 2-h weekly classes, then three 2-h classes every 2 weeks, followed by four 1.5 -h classes every 2 months. One group leader instructed LCK participants to eat an ad libitum very low-carbohydrate, likely ketogenic diet, by reducing their carbohydrate intake to between 20-50 g of carbohydrates (excluding fiber) a day. We gave them a goal of achieving a blood ketone (betahydroxybutyrate) level of between 0.5 and 3 millimolar (mmol), as measured twice a week for the first several months at home. A different group leader instructed the MCCR participants to follow an MCCR diet in which $45-50 \%$ of their calories were to be derived from carbohydrates. We also instructed them to lower their fat consumption and eat 500 fewer kilocalories (kcal) per day than their calculated maintenance needs to reduce weight.

Starting in week 6, group leaders taught participants in both groups about the importance of sleep and exercise for type 2 diabetes and encouraged them to increase both, if needed. A third group leader also taught all participants supportive behavioral adherence strategies aimed at increasing positive affect ${ }^{12}$ and mindful eating ${ }^{13}$, in order to increase intervention adherence. See our publication of the 3-month outcomes from this study for full method specifications $^{11}$.

\section{Assessments}

We obtained a fasting blood specimen and measured participants' $\mathrm{HbA}_{1 \mathrm{c}}$, lipids, fasting glucose and insulin, and C-reactive protein. Tests were performed by a commercial CLIA-certified laboratory (Quest Diagnostics, Madison, NJ), which was unaware of the study design or assigned conditions. Study staff measured weight and blood pressure during in-person visits. We estimated insulin resistance using homeostatic model assessment (HOMA2-IR ${ }^{14}$. Participants recorded their food using the Automated Self-Administered 24-h Dietary Recall $(\text { ASA24) })^{15}$. At baseline and 3, 6, and 12 months after baseline we asked participants to report what they ate during one day.

\section{Statistical analysis}

The statistical analyses were performed using Stata, IC software version 14.1 (StataCorp LP, College Station, TX, USA). The outcomes were continuously scaled and were analyzed with parametric statistical techniques. Several outcomes required log transformation prior to analysis and exclusion of a few influential outliers. Participants' repeated measures outcomes were submitted to separate mixed-effects linear regression analyses with fixed effect terms comparing baseline to each of the three subsequent time points after baseline, the main effect for group, and the simple interaction effects comparing the relative change by group at each post-baseline assessment, relative to baseline. Random y-intercept terms were included to accommodate for the repeated measures experimental design. For all results involving dichotomous outcomes, we used a two-tailed Fisher exact test to assess significance. We tested the difference between groups for percent weight lost with an independent samples $t$-test for the change within each group. As this was an exploratory pilot trial, the trial was not powered.

\section{Results}

\section{Recruitment and participation}

We enrolled and randomized 34 participants to the LCK $(n=16)$ or MCCR $(n=18)$ group (Supplementary Fig. 1$)$. Retention did not differ by intervention group: 12-month retention for the LCK group was 14/16 (87.5\%) and 15/18 (83.3\%) for the MCCR group $(p=1.000)$, with an average of $85.3 \%$ participants retained (Supplementary Table 1).

\section{Dietary assessment}

Compared to the MCCR group, the LCK group reported consuming fewer non-fiber grams of carbohydrates $(6$ and 12 months), more grams of fat (6 and 12 months), and more grams of protein (12 months), but not a different number of calories per day (Table 1).

$\mathrm{HbA}_{1 \mathrm{c}}$

At 12 months, participants in the LCK group reduced their $\mathrm{HbA}_{1 \mathrm{c}}$ levels more than participants in MCCR group (Table 1, Fig. 1). In the LCK versus the MCCR group, at both 6 and 12 months, more than twice the percentage of participants who began with an $\mathrm{HbA}_{1 \mathrm{c}}$ at or above $6.5 \%$, the cutoff for type 2 diabetes, ended below this level. However, this result was only significant at 6 months (Supplementary Table 2).

\section{Body weight and other health outcomes}

At 12 months, participants in the LCK group lost more weight and lowered their BMI more than participants in 
Table 1 Estimated marginal mean $(E M M) \pm 95 \% \mathrm{Cl}$ at baseline to 6 and 12 months

\begin{tabular}{|c|c|c|c|}
\hline Outcomes & LCK group, EMM (95\% Cl) & MCCR, EMM (95\% Cl) & $\begin{array}{l}P \text { value (interactions comparing group } \\
\text { differences in changes relative to baseline) }\end{array}$ \\
\hline \multicolumn{4}{|l|}{$\mathrm{HbA}_{1 \mathrm{c}}(\%)$} \\
\hline Baseline & $6.6(6.3,6.9)$ & $6.9(6.6,7.2)$ & \\
\hline 6 months & $6.0(5.7,6.3)$ & $6.7(6.4,6.9)$ & .001 \\
\hline 12 months & $6.1(5.8,6.4)$ & $6.7(6.4,7.0)$ & .007 \\
\hline \multicolumn{4}{|c|}{ Body mass index $\left(\mathrm{kg} / \mathrm{m}^{2}\right)$} \\
\hline Baseline & $35.9(32.5,39.2)$ & $36.9(33.7,40.1)$ & \\
\hline 6 months & $33.7(30.3,37.1)$ & $36.0(32.9,39.2)$ & .001 \\
\hline 12 months & $33.3(29.9,36.7)$ & $36.0(32.8,39.2)$ & $<.001$ \\
\hline \multicolumn{4}{|c|}{ Body weight (kg) } \\
\hline Baseline & $99.9(88.4,111.5)$ & $97.5(86.6,108.3)$ & \\
\hline 6 months & $93.8(82.3,105.3)$ & $95.8(84.9,106.6)$ & $<.001$ \\
\hline 12 months & $92.0(80.5,103.6)$ & $95.8(84.9,106.6)$ & $<.001$ \\
\hline \multicolumn{4}{|c|}{ Triglycerides (mg/dL) } \\
\hline Baseline & $102.6(81.8,123.4)$ & $158.9(128.8,189.1)$ & \\
\hline 6 months & $86.2(68.6,103.7)$ & $143.2(115.6,170.9)$ & .48 \\
\hline 12 months & $92.7(73.6,111.7)$ & $173.4(138.1,208.7)$ & .08 \\
\hline \multicolumn{4}{|c|}{ HDL cholesterol (mg/dL) } \\
\hline Baseline & $48.4(42.6,54.2)$ & $45.8(40.6,51.0)$ & \\
\hline 6 months & $51.9(45.7,58.2)$ & $48.1(42.5,53.6)$ & .58 \\
\hline 12 months & $53.3(46.8,59.8)$ & $48.9(43.3,54.5)$ & .45 \\
\hline \multicolumn{4}{|c|}{ LDL cholesterol (mg/dL) } \\
\hline baseline & $88.7(76.3,101.1)$ & $98.1(86.4,109.8)$ & \\
\hline 6 months & $97.9(85.4,110.5)$ & $88.1(76.0,100.1)$ & .003 \\
\hline 12 months & $95.6(82.3,108.9)$ & $96.1(83.7,108.5)$ & .20 \\
\hline \multicolumn{4}{|c|}{ Triglycerides (mg/dL)/HDL cholesterol (mg/dL) } \\
\hline baseline & $2.2(1.7,2.7)$ & $3.5(2.7,4.2)$ & \\
\hline 6 months & $1.7(1.3,2.1)$ & $3.0(2.4,3.7)$ & .18 \\
\hline 12 months & $1.7(1.3,2.1)$ & $3.6(2.8,4.5)$ & .022 \\
\hline \multicolumn{4}{|c|}{ C-reactive protein (mg/dL) } \\
\hline Baseline & $4.2(2.1,6.4)$ & $3.3(1.7,4.9)$ & \\
\hline 6 months & $2.9(1.4,4.5)$ & $2.5(1.3,3.6)$ & .69 \\
\hline 12 months & $3.0(1.5,4.6)$ & $2.3(1.2,3.4)$ & .85 \\
\hline \multicolumn{4}{|c|}{ Fasting insulin $(\mu \mathrm{l} \mathrm{U} / \mathrm{mL})$} \\
\hline Baseline & $8.7(6.0,11.3)$ & $8.9(6.4,11.5)$ & \\
\hline 6 months & $8.9(6.2,11.6)$ & $11.9(8.4,15.3)$ & .09 \\
\hline 12 months & $9.1(6.3,12.0)$ & $10.1(7.1,13.0)$ & .66 \\
\hline \multicolumn{4}{|l|}{ HOMA2-IR } \\
\hline Baseline & $1.0(0.7,1.4)$ & $1.1(0.8,1.5)$ & \\
\hline
\end{tabular}


Table 1 continued

\begin{tabular}{|c|c|c|c|}
\hline Outcomes & LCK group, EMM $(95 \% \mathrm{Cl})$ & MCCR, EMM $(95 \% \mathrm{Cl})$ & $\begin{array}{l}P \text { value (interactions comparing group } \\
\text { differences in changes relative to baseline) }\end{array}$ \\
\hline 6 months & $1.2(0.8,1.5)$ & $1.4(1.0,1.9)$ & .38 \\
\hline 12 months & $1.0(0.7,1.3)$ & $1.2(0.9,1.6)$ & .51 \\
\hline \multicolumn{4}{|c|}{ Diastolic blood pressure $(\mathrm{mm} \mathrm{Hg})$} \\
\hline Baseline & $77.1(74.0,80.3)$ & $81.1(78.2,84.1)$ & \\
\hline 6 months & $77.1(74.0,80.1)$ & $80.8(77.9,83.7)$ & .90 \\
\hline 12 months & $75.6(72.5,78.8)$ & $78.4(75.5,81.4)$ & .57 \\
\hline \multicolumn{4}{|c|}{ Systolic blood pressure $(\mathrm{mm} \mathrm{Hg})$} \\
\hline Baseline & $127.1(121.9,132.3)$ & $129.2(124.6,133.7)$ & \\
\hline 6 months & $130.7(125.7,135.7)$ & $130.4(125.6,135.1)$ & .47 \\
\hline 12 months & $130.3(125.2,135.4)$ & $127.5(122.7,132.4)$ & .15 \\
\hline \multicolumn{4}{|c|}{ Total kilocalories } \\
\hline Baseline & $1954.1(1617.7,2290.5)$ & $2063.1(1733.5,2392.7)$ & \\
\hline 6 months & $1589.4(1315.1,1863.7)$ & $1483.5(1236.7,1730.3)$ & .33 \\
\hline 12 months & $1534.8(1255.5,1814.0)$ & $1681.1(1387.4,1974.9)$ & .78 \\
\hline \multicolumn{4}{|c|}{ Total grams of non-fiber carbohydrates } \\
\hline Baseline & $176.2(143.9,208.6)$ & $184.4(152.7,216.2)$ & \\
\hline 6 months & $44.1(27.4,60.8)$ & $160.7(131.0,190.4)$ & $<.001$ \\
\hline 12 months & $73.7(51.5,96.0)$ & $149.8(119.4,180.2)$ & .002 \\
\hline \multicolumn{4}{|c|}{ Total grams of fat } \\
\hline Baseline & $79.2(59.8,98.6)$ & $86.3(66.8,105.8)$ & \\
\hline 6 months & $101.4(76.5,126.3)$ & $55.8(42.6,69.0)$ & .001 \\
\hline 12 months & $105.4(79.4,131.3)$ & $75.4(56.5,94.2)$ & .037 \\
\hline \multicolumn{4}{|c|}{ Total grams of protein } \\
\hline Baseline & $82.7(65.1,100.4)$ & $91.4(72.6,110.3)$ & \\
\hline 6 months & $92.2(72.4,112.0)$ & $82.7(65.9,99.5)$ & .12 \\
\hline 12 months & $97.6(76.3,118.9)$ & $68.8(53.8,83.9)$ & .002 \\
\hline
\end{tabular}

Data are estimated marginal means and $95 \%$ confidence intervals by linear mixed-effects model analysis

the MCCR group (Table 1, Supplementary Fig. 2). On average, at 12 months participants in the LCK group lost $8.3 \%$ of body weight, whereas the MCCR group lost $3.8 \%$ (Supplementary Table 2).

At 6 months, LDL cholesterol increased more in the LCK group compared to the MCCR group, although at 12 months the groups no longer significantly differed (Table 1). At 6 months, change in the ratio of triglycerides to HDL cholesterol did not significantly differ across groups, although at 12 months this ratio had decreased more in the LCK group compared to the MCCR group (Table 1). Other biological outcomes did not differ significantly across groups (Table 1 ).

\section{Diabetes medications}

Participants in the LCK group reduced their use of some diabetes-related medications more than participants in the MCCR group. Of ten participants who reported taking sulfonylureas or dipeptidyl peptidase-4 inhibitors before the intervention, all six participants assigned to the LCK group discontinued these medications by 12 months postbaseline (at the request of the study physicians, based on the study protocol), compared with none of the four participants in the MCCR group ( $p=.005$, Fischer's exact test). Two participants in the MCCR group began taking these medications, whereas no participants in the LCK group did so. Of 22 participants who reported taking 


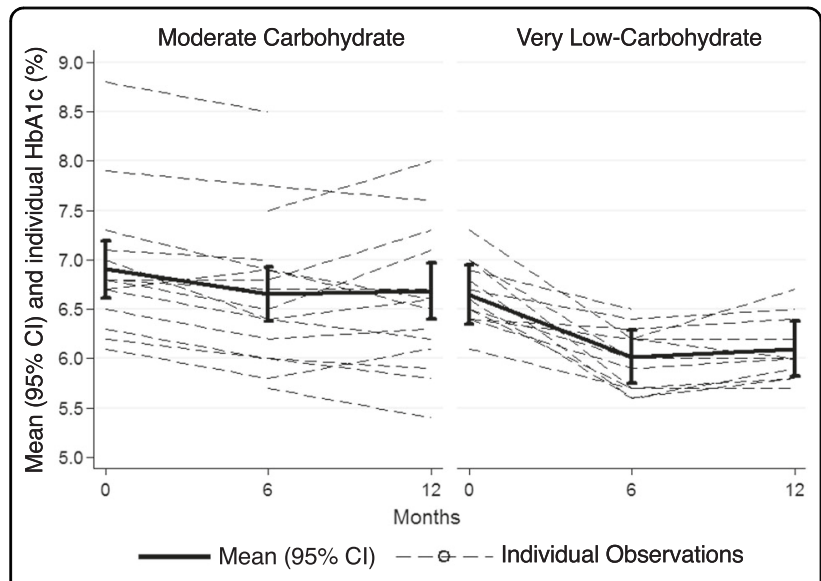

Fig. 1 Mean and individual $\mathrm{HbA}_{1 \mathrm{c}}$ for the two groups at baseline and at 6 and 12 months. Bars represent standard 95\% confidence intervals of the mean. Dashed lines reflect individual participant observations; darker lines represent each group mean

metformin before the intervention, $3 / 10$ in the in the LCK group discontinued the medication, compared with $0 / 12$ in the MCCR group $(p=.08$, Fisher's exact test). In addition, in the LCK group 1 person increased their dose of metformin and in the MCCR group 2 people decreased their metformin, with none of these changes being significantly different between groups $(p>.3)$.

\section{Discussion}

Twelve months after baseline, participants in the LCK group evidenced greater reductions in each $\mathrm{HbA}_{1 \mathrm{c}}$ and weight than did participants in the MCCR group. In addition, the greater reductions in $\mathrm{HbA}_{1 \mathrm{c}}$ in the LCK group occurred despite greater reductions in glucoselowering medications. A strength of our trial was that few participants dropped out after 12 months. However, the ability to generalize from this study is limited by its relatively small size, which did not allow us to perform subgroup analyses.

At 6 months, we noted an increase in LDL cholesterol in the LCK group compared to the MCCR group. This difference was no longer significant at 12 months. This may raise some concerns about the long-term effects of such a diet on cardiovascular disease. Recent research suggests that the correlation of LDL to cardiovascular risk varies based on particle size ${ }^{16,17}$, and that low-carbohydrate ketogenic diets tend to increase LDL particle size ${ }^{18}$, which suggests that the increase in total LDL may not be accompanied by increased cardiovascular risk. However, we did find that the ratio of triglycerides to HDL, which predicts coronary disease ${ }^{19}$, decreased in the LCK group compared to the MCCR group, suggesting that the very low-carbohydrate ketogenic diet may have certain benefits on lipid profiles. In addition, no participants experienced diabetic ketoacidosis, a condition that occurs when ketone production is unregulated, with blood ketone levels surpassing $25 \mathrm{mM}$, with concurrent high blood glucose levels (above $250 \mathrm{mg} / \mathrm{dL}$ ), an unlikely scenario in this research ${ }^{20}$.

Our trial had good retention and fair dietary adherence, which may have been due to the novel supportive psychological strategies (positive affect and mindful eating). Future trials could explicitly test the hypothesis that these strategies may improve retention and adherence rates. Moreover, because both groups also received lifestyle recommendations (physical activity and sleep), we are limited in understanding how the diets may have influenced outcomes independent of the other suggested changes. In future trials, if researchers randomized participants to different combinations of lifestyle and psychological recommendations, this could clarify and optimize the most helpful adjunctive advice and support.

The results suggest that adults with prediabetes or noninsulin-dependent type 2 diabetes may be able to improve glycemic control with less medication by following an ad libitum very low-carbohydrate ketogenic diet compared to a moderate-carbohydrate, calorie-restricted low-fat diet. Additional research should examine both clinical outcomes and adherence beyond 12 months.

\section{Acknowledgements}

Thanks very much to Michael Hall for his help with running the in-person study, Carley Hauck for teaching the positive affect and mindful eating skills, and to our dedicated participants. The research was supported by a grant from the William K. Bowes, Jr. Foundation and the Mount Zion Health Fund. Laura Saslow and Ashley E. Mason were supported by National Institutes of Health grant T32AT003997 from the National Center for Complementary and Integrative Health. Laura Saslow was also supported by a K01 from the National Institute of Diabetes and Digestive and Kidney Diseases (DK107456). Ashley E. Mason was also supported by a K23 from the National Heart, Lung, and Blood Institute (HL133442). Judith Moskowitz was supported by National Institutes of Health grant K24 MH093225 from the National Institute of Mental Health. Frederick Hecht was supported by National Institutes of Health grant K24 AT007827 from National Center for Complementary and Integrative Health. The funders had no role in study design, data collection and analysis, decision to publish, or preparation of the manuscript.

\section{Author details}

${ }^{1}$ University of Michigan, Ann Arbor, MI, USA. ${ }^{2}$ San Francisco State University, San Francisco, CA, USA. ${ }^{3}$ Northwestern University, Evanston, IL, USA. ${ }^{4}$ University of California, San Francisco, CA, USA. ${ }^{5}$ Virta Health, San Francisco, CA 94105, USA. ${ }^{6}$ UCSF Benioff Children's Hospital Oakland, Oakland, CA, USA

\section{Competing interests}

Stephen Phinney is a paid member of the Atkins Scientific Advisory Board, a founder of Virta Health, and has authored books on low-carbohydrate, high fat diets: New Atkins and You, The Art and Science of Low Carbohydrate Living, and The Art and Science of Low Carbohydrate Performance. Frederick Hecht is on the Scientific Advisory Board for Virta Health. The other authors declare no competing financial interests.

\section{Supplementary information}

The online version of this article (https://doi.org/10.1038/s41387-017-0006-9) contains supplementary material. 
Received: 18 June 2017 Revised: 28 September 2017 Accepted: 18 October 2017

Published online: 21 December 2017

\section{References}

1. Feinman, R. D. et al. Dietary carbohydrate restriction as the first approach in diabetes management: critical review and evidence base. Nutrition 31, 1-13 (2015).

2. Volek, J. S. et al. Carbohydrate restriction has a more favorable impact on the metabolic syndrome than a low fat diet. Lipids 44, 297-309 (2009).

3. Ruskin, D. N. \& Masino, S. A. The nervous system and metabolic dysregulation: emerging evidence converges on ketogenic diet therapy. Front Neurosci. 6, 33 (2012).

4. Dashti, H. M. et al. Beneficial effects of ketogenic diet in obese diabetic subjects. Mol. Cell. Biochem. 302, 249-256 (2007).

5. Boden, G., Sargrad, K., Homko, C., Mozzoli, M. \& Stein, T. P. Effect of a lowcarbohydrate diet on appetite, blood glucose levels, and insulin resistance in obese patients with type 2 diabetes. Ann. Intern. Med. 142, 403-411 (2005).

6. Saslow, L. R. et al. An online intervention comparing a very low-carbohydrate ketogenic diet and lifestyle recommendations versus a plate method diet in overweight individuals with type 2 diabetes: a randomized controlled trial. J. Med. Internet Res. 19, e36, https://doi.org/10.2196/jmir.5806 (2017).

7. Hussain, T. A. et al. Effect of low-calorie versus low-carbohydrate ketogenic diet in type 2 diabetes. Nutrition 28, 1016-1021 (2012).

8. Davis, N. J. et al. Comparative study of the effects of a 1-year dietary intervention of a low-carbohydrate diet versus a low-fat diet on weight and glycemic control in type 2 diabetes. Diabetes Care 32, 1147-1152 (2009).

9. Tay, J. et al. Comparison of low-and high-carbohydrate diets for type 2 diabetes management: a randomized trial. Am. J. Clin. Nutr. 102, 780-790 (2015).
10. Westman, E. C. et al. The effect of a low-carbohydrate, ketogenic diet versus a low-glycemic index diet on glycemic control in type 2 diabetes mellitus. Nutr. Metab. 5, 36 (2008).

11. Saslow, L. R. et al. A randomized pilot trial of a moderate carbohydrate diet compared to a very low carbohydrate diet in overweight or obese individuals with Type 2 diabetes mellitus or prediabetes. Plos One 9, e91027 (2014).

12. Moskowitz, J. T. et al. A positive affect intervention for people experiencing health-related stress: development and non-randomized pilot test. J. Health Psychol. 17, 676-692 (2011).

13. Daubenmier, J. et al. Mindfulness intervention for stress eating to reduce cortisol and abdominal fat among overweight and obese women: an exploratory randomized controlled study. J. Obes. 2011, 651936 (2011).

14. Wallace, T. M., Levy, J. C. \& Matthews, D. R. Use and abuse of HOMA modeling. Diabetes Care 27, 1487-1495 (2004).

15. Subar, A. F. et al. The automated self-administered 24-hour dietary recall (ASA24): a resource for researchers, clinicians, and educators from the national cancer institute. J. Acad. Nutr. Diet. 112, 1134-1137 (2012).

16. Austin, M. A. et al. Low-density lipoprotein subclass patterns and risk of myocardial infarction. JAMA 260, 1917-1921 (1988).

17. Davidson, M. H. et al. Clinical utility of inflammatory markers and advanced lipoprotein testing: advice from an expert panel of lipid specialists. J. Clin. Lipidol. 5, 338-367 (2011).

18. Gerber, P. A. \& Berneis, K. Regulation of low-density lipoprotein subfractions by carbohydrates. Curr. Opin. Clin. Nutr. Metab. Care 15, 381-385 (2012).

19. da Luz, P. L., Favarato, D., Junior, J. R. F.-N., Lemos, P. \& Chagas, A. C. P. High ratio of triglycerides to hdl-cholesterol predicts extensive coronary disease. Clin. (Sao Paulo, Braz.). 63, 427-432, https://doi.org/10.1590/S180759322008000400003 (2008).

20. Manninen, A. H. Metabolic Effects of the very-low-carbohydrate diets: misunderstood villains of human metabolism. J. Int. Soc. Sports Nutr. 1, 7-11 (2004). 